\title{
DWARF NOVA OUTBURSTS AND SUPEROUTBURSTS
}

\author{
J. SMAK \\ N. Copernicus Astronomical Center, \\ Polish Academy of Sciences, \\ Bartycka 18, 00-716 Warsaw, Poland \\ e-mail: jis@camk.edu.pl
}

\section{The viscosity parameter $\alpha$}

Dwarf nova outbursts provide an almost unique opportunity of getting an insight into the nature of viscosity in accretion disks or, within the $\alpha$-disk approach, of putting some constraints on $\alpha$. In particular, the strong dependence of the viscous time-scale on viscosity itself permits us to estimate $\alpha$ almost directly from the observed time-scales. In the case of the hot branch of the $\Sigma-T_{\mathrm{e}}$ relation, the most reliable estimates $\left(\alpha_{\mathrm{hot}}\right)$ are based on the rate of decline following the dwarf nova outburst. From a comparison with model light curves calculated with different $\alpha$ s one gets: $\alpha_{\text {hot }} \approx 0.2$ (e.g. Smak 1984b). An independent, but much cruder, estimate can be obtained from the widths of normal outbursts, by assuming that the duration of an outburst represents the travel time of an instability wave across the disk. The result is similar: $\alpha_{\text {hot }} \approx 0.2$ (Gicger 1987).

In the case of the cool branch of the $\Sigma-T_{\mathrm{e}}$ relation, estimates of $\alpha$ $\left(\alpha_{\text {cool }}\right)$ can be obtained from theoretical relation(s), based on models of dwarf nova outbursts, which predict the observed length of the outburst cycle to be a function of $\alpha_{\text {cool }}$, the mass transfer rate and other system parameters (see Cannizzo 1993). This was done by Livio \& Spruit (1991) who applied such a relation from Cannizzo, Shafter \& Wheeler (1988) to a large number of dwarf novae with reliable system parameters (Warner 1987). For the purpose of this presentation, these estimates have been repeated with a somewhat updated set of data (but with no attempt to update the theoretical part with more recent model calculations). The results are shown in Fig. 1 as squares. Another set of estimates of $\alpha_{\text {cool }}$ can be obtained from much cruder considerations, applicable to type B outbursts (Smak 1993, 


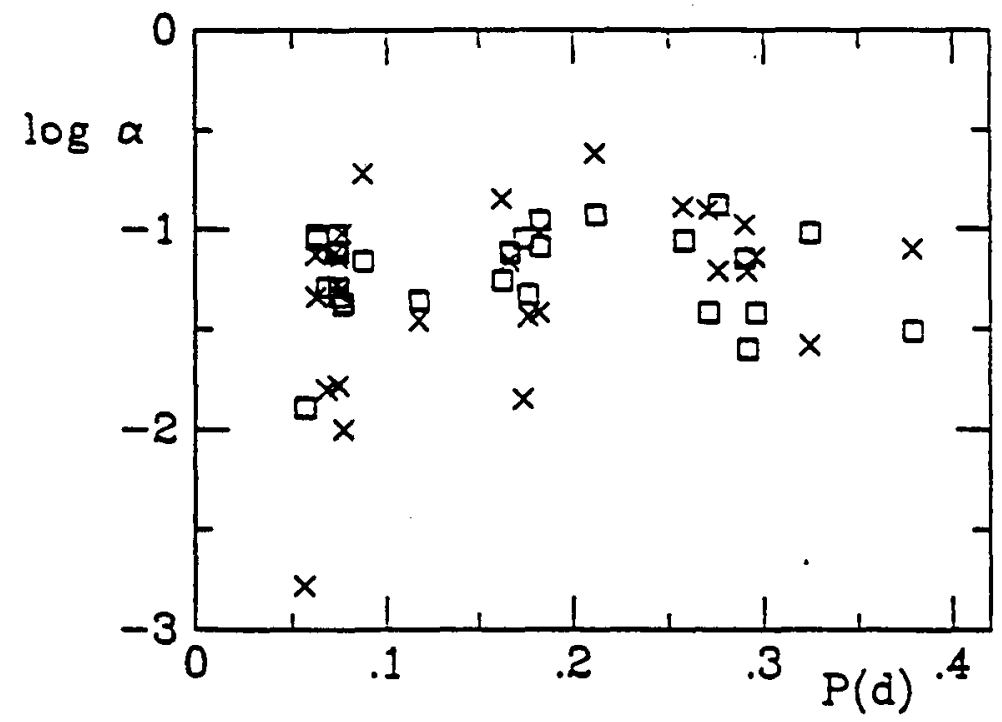

Figure 1. Viscosity parameter $\alpha_{\text {cool }}$, estimated from a sample of dwarf novae, plotted against the orbital period. See text for details.

equation 23); note that, in the case of type $A$ outbursts, such estimates give only an upper limit to $\alpha$. The results are shown in Fig. 1 as crosses.

The following points can be made after inspecting Fig. 1: (i) the two types of estimates agree reasonably well; (ii) there is no correlation with the orbital period (but there might be a weak correlation with the mass ratio; Livio \& Spruit 1991); (iii) the 'mean' value of $\alpha_{\text {cool }}$ is, roughly, about 0.05 ; (iv) the scatter does not exceed a factor of 2 or 3 and must be, at least partly, due to uncertainties involved, so that no firm conclusion about its intrinsic value can be made.

With estimates of $\alpha_{\text {hot }}$ and $\alpha_{\text {cool }}$, as presented above, we get $\alpha_{\text {hot }} / \alpha_{\text {cool }} \approx$ 4 , a value which is independently suggested (in fact: strongly required) by model calculations for dwarf nova outbursts (see Cannizzo 1993).

The picture presented so far, however, is not complete. It does not include dwarf novae belonging to the SU UMa subtype, which show only the superoutbursts (group C of Vogt 1993) with very long superoutburst cycles and very large superoutburst amplitudes (the TOADs, Howell, Szkody \& Cannizzo 1995). The very long outburst cycles, lasting years or decades, clearly imply that either the viscosity $\left(\alpha_{\text {cool }}\right)$, or the mass transfer rate (or both) must be much lower than in the case of other dwarf novae. Concerning the mass transfer rate we must note that there is a natural lower limit set by the effects of the gravitational radiation. These qualitative considerations are confirmed by results available for WZ Sge, the most extreme and the best documented member of the group: the mass transfer rate is indeed very 
low $\left(\dot{M} \approx 10^{15} \mathrm{~g} \mathrm{~s}^{-1}\right)$ and consistent with theoretical predictions (Paczyński \& Sienkiewicz 1983), while the viscosity in its disk during quiescence is 2 or 3 orders of magnitude lower than in other dwarf novae (Osaki 1995; Smak 1993).

It is not clear at present whether other objects of this group have also systematically lower viscosity or, more likely, form a continous transition from 'normal' dwarf novae to the extreme case of WZ Sge. In the latter case, we could probably expect $\alpha_{\text {cool }}$ to be correlated with the length of the superoutburst cycle (note, for example, that the lowest point in Fig. 1 is SW UMa with a superoutburst period of $570 \mathrm{~d}$ ). But, if so, the main question would be: what are the basic system parameters related to, or responsible for, those lower values of $\alpha_{\text {cool }}$ ? An answer to that question might be crucial for our understanding of the nature of viscosity. Unfortunately, except for WZ Sge, reliable determinations of basic system parameters are not available for members of this group and this appears to be one of the most important and urgent goals for observers.

\section{Two types of dwarf nova outbursts}

Models of dwarf nova outbursts predicted two types of outbursts: type A, when the thermal instability occurs first in the outer parts of the disk and propagates inward and type B, when it begins in the inner parts and propagates outward. Generally, type B outbursts occur at lower mass transfer rates. There are now 15 dwarf novae with positively identified outburst types (Smak 1987; Warner 1995, Table 3.6). Using this sample we can conclude that:

(i) both types of outbursts are represented among dwarf novae of the U Gem subtype;

(ii) dwarf novae of the SU UMa subtype show only type A outbursts. This implies that the lower limit to the mass transfer rate set by the effects of the gravitational radiation is sufficiently high to inhibit the occurence of type B outbursts;

(iii) dwarf novae of the Z Cam subtype show only type B outbursts. This implies that the mass transfer rate during the dwarf nova phase is considerably lower than the mean mass transfer rate.

\section{Effects of dwarf nova outbursts}

\subsection{HEATING OF THE WHITE DWARF}

The first dwarf nova for which clear photometric evidence for substantial heating of the white dwarf during outburst and its subsequent cooling during quiescence was available some 30 years ago, namely U Gem (Paczyński 
1965). Thanks to the effort of many observers and particularly, the availability of the Hubble Space Telescope, we now have very extensive photometric and spectroscopic coverage for several dwarf novae (OY Car: Marsh, Horne \& Cheng 1993; Z Cha: Wood et al. 1993; U Gem: Huang, Sion \& Szkody 1995; WZ Sge: Smak 1993, Sparks et al. 1993), including their outbursts and superoutbursts.

Theoretical interpretation of these data (e.g. Sparks et al. 1993; Sparks 1996) gives us deeper insight into the structure of the boundary layer and of the outer layers of the accreting white dwarf. It may be worth noting here that, in the case of non-magnetic CVs, the heating of the white dwarf and the accretion onto its surface both occur in the equatorial regions (while in the case of magnetic CVs this happens in the polar caps). Better understanding of these phenomena is needed for studies involving meridional circulation and mixing, i.e. processes of crucial importance for the theory of nova outbursts.

\subsection{HEATING OF THE SECONDARY}

There is ample observational evidence showing this effect. The best documented examples are: SS Cyg (Hessman et al. 1984; Echevarria et al. 1989) and IP Peg (Martin et al. 1989; Marsh \& Horne 1990), while the most dramatic case was probably the 1978 superoutburst of WZ Sge, during which the temperature of its secondary exceeded $10000 \mathrm{~K}$ (Smak 1993).

With this evidence being now available and likely to be extended by future observations, the problem is ripe for theoretical studies (e.g. Sarna 1990; Brett \& Smith 1993; Hameury, King \& Lasota 1993). One of the most important questions here concerns the mechanism(s) responsible for the resulting enhanced mass outflow from the surface of the secondary (see next Section). Heating by radiation affects directly only those parts of the star's hemisphere facing the primary which are not shielded by the disk. Its equatorial parts, including the vicinity of $L_{1}$, are not irradiated. The question is then: does the heating affect indirectly local conditions around $L_{1}$ in a sufficient degree to increase significantly the rate of outflow, or should we rather look for another mechanism?

\subsection{ENHANCED MASS TRANSFER RATE}

Modulation of the mass tranfer rate, due to the time dependent heating of the secondary, was an essential ingredient of models proposed to explain the Z Cam behavior (Meyer \& Meyer-Hofmeister 1983) and the superoutbursts of SU UMa stars (Osaki 1985). At that time the only observational evidence for a substantial increase of $\dot{M}$ during outbursts was due to Vogt (1983), who found that the hot spot in VW Hyi becomes considerably brighter, 


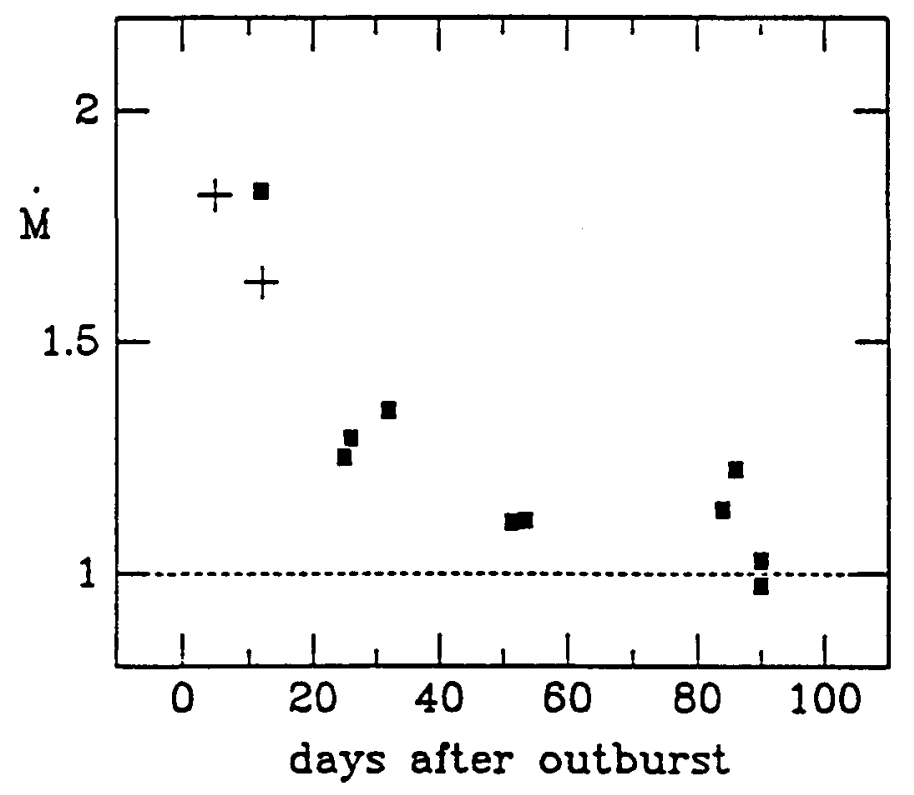

Figure 2. Variations in the mass transfer rate in U Gem, normalized to $\dot{M}=1$ just before outburst (from Smak 1995). Filled squares are based on luminosities of the hot spot measured during its eclipse. The crosses - on luminosities corresponding to the amplitude of the hump.

indicating an increase in $\dot{M}$, during those outbursts which occur less than $40 \mathrm{~d}$ before the next superoutburst.

We note in passing that, while the luminosity of the hot spot is an obvious measure of the mass transfer rate, it must be remembered that it also depends on the impact parameter, i.e. on the relative velocity of collision between the stream and the outer parts of the disk. When the radius of the disk varies within the outburst cycle (Sect. 3.4), the effect of such variations should be taken into account in the determination of $\dot{M}$.

Possible evidence for an enhanced $\dot{M}$ during a superoutburst came from a single eclipse observed early in a superoutburst of $\mathrm{Z}$ Cha (Warner \& O'Donoghue 1988), the shape of which could be interpreted as indicative of a very bright hot spot. An important point here is that, generally, it is difficult to separate the hot spot contribution from superhumps and photometric effects connected with the heating of the secondary. Furthermore, if superoutbursts are due to an enhanced mass transfer, then it is only during the initial phase of presumably rapid increase of $\dot{M}$ that the hot spot can be relatively bright. Consequently, it is very difficult to identify possible effects of enhanced $\dot{M}$ during superoutbursts and the absence of more convincing observational evidence cannot be treated as proof that such an enhancement occurs only sporadically, or does not occur at all. 
Good evidence is now available (Smak 1995) for modulated mass transfer rate during normal outburst cycles in two dwarf novae: U Gem (Fig. 2) and $\mathrm{Z}$ Cha. In both of them the mass transfer rate increases during outbursts by a factor of 2 , with the subsequent decline in U Gem taking, roughly, one-half of its outburst cycle. We can conclude that the modulation of the mass transfer rate within the outburst cycle is probably a common phenomenon among dwarf novae.

\subsection{DISK RADIUS VARIATIONS}

Variations of the disk radius during the dwarf nova cycle are best documented in the case of two eclipsing dwarf novae: U Gem (Smak 1984a) and IP Peg (Wood et al. 1989; Wolf et al. 1993). In both cases the radius increases during outburst by about $30 \ldots 40 \%$, and then decreases slowly during quiescence; in both cases variations repeat quite well from cycle to cycle. Detailed comparison between model-predicted and observed disk radius variations can serve not only as a test for models of dwarf nova outbursts but may also permit an independent estimate of viscosity. It is clear that, in order to make such a comparison more meaningful, the models should include the effect of variable $\dot{M}$ within the dwarf nova cycle.

Data are also available for two eclipsing dwarf novae of the SU UMa subtype. In the case of Z Cha (O'Donoghue 1986; Zoła 1989) disk radius variations, when plotted against time since the last outburst, show considerable scatter due, in part, to the poor repetition from cycle to cycle. But when the phase of the outburst cycle is used instead of time, the characteristic pattern, similar to that in U Gem, becomes visible. In the case of OY Car (Fig. 3) no regular variations of the type described above can be seen in either of the two plots.

The evidence available for $\mathrm{Z}$ Cha and, particularly, for OY Car should not be considered as disappointing. On the contrary, it may help us look for effects which, so far, have not been included in our considerations. The most obvious difference between $\mathrm{Z}$ Cha and OY Car on the one side, and U Gem and IP Peg on the other, is that the former two, as belonging to the SU UMa subtype, show superoutbursts. To that end, simple models of superoutbursts, involving either the enhanced mass transfer, or the tidal instability of an eccentric disk, predict very regular behavior of $R_{\mathrm{d}}$, of the type observed in U Gem (Osaki 1989, Smak 1991). Clearly then, the mechanism of superoutbursts is likely to include an additional factor responsible for observed irregularities. The case of $\mathrm{Z}$ Cha may be particularly instructive in this respect: it suggests that the length of the outburst cycles within the supercycle and $R_{\mathrm{d}}$ are likely to be controlled by the same unknown factor. At the same time the difference between $\mathrm{Z}$ Cha and OY Car may 


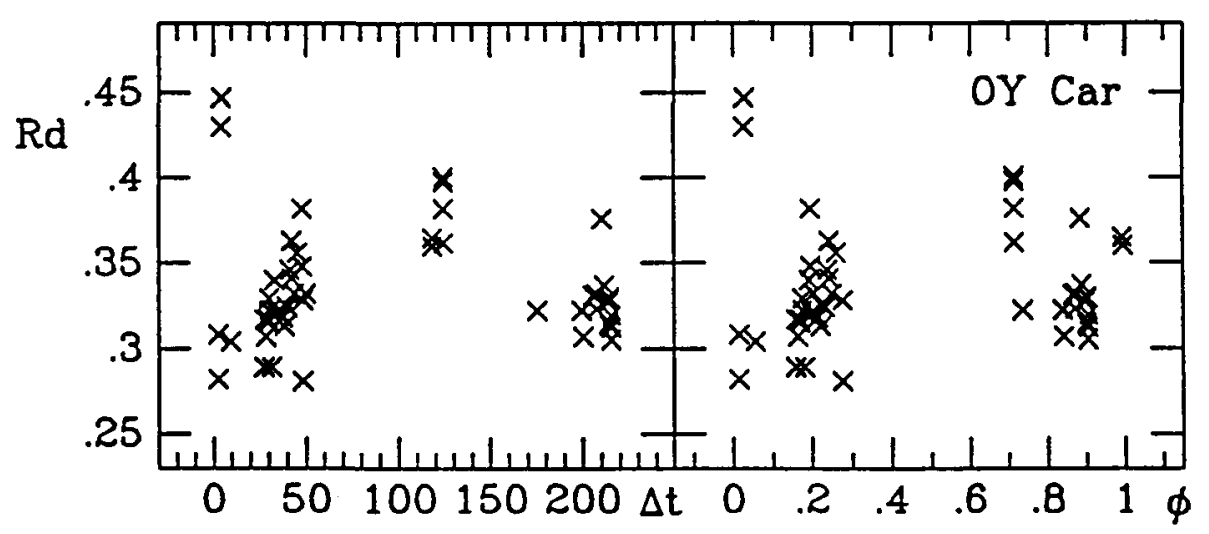

Figure 3. Disk radius variations in OY Car plotted against time $\Delta t$ since the last outburst (left) and the phase $\phi$ of the outburst cycle (right). All data available in the literature are used in these diagrams.

somehow reflect their different outburst/superoutburst patterns: in OY Car there are fewer normal outbursts within each superoutburst cycle.

\section{The superoutbursts}

\subsection{THE ENHANCED MASS TRANSFER (EMT) MODEL}

This is the model by Osaki (1985) involving a feedback between the accretion rate, the irradiation of the secondary, and the mass transfer rate, which - under certain conditions - may result in an instability leading to a limit cycle behavior. The evidence now available (Sect. 3.3 ) clearly shows that, indeed, the mass transfer rate undergoes enhancement during normal outbursts and, very likely, during superoutbursts.

Osaki (1985) proposed also a model for superhumps, combining the EMT model with that of a slowly precessing eccentric disk (Vogt 1981). In this model the irradiation of the secondary was modulated with the synodic period due to varying extent of the eccentric disk's shadow, causing the mass transfer rate and, consequently, the luminosity of the hot spot, to vary with that same period. This model was substantially modified by Mineshige (1988) who included the effects of heating of the outer parts of the disk thereby offering a potential explanation for the variable azimuthal distribution and extension of the superhump source (e.g. Warner \& O'Donoghue 1988). 


\subsection{THE THERMAL AND TIDAL INSTABILITY (TTI) MODEL}

This model (Osaki 1989) combines the effects of the thermal instability responsible for dwarf nova outbursts, with the tidal instability which occurs due to the 3:1 resonance - for large disks in systems with $q<q_{\text {crit }} \approx 0.25$, and leads to a development of an eccentric, precessing disk (Whitehurst 1988; Hirose \& Osaki 1990; Whitehurst \& King 1991; Osaki, Hirose \& Ichikawa 1993).

This model successfully reproduces light curves of ordinary SU UMa stars with normal outbursts and superoutbursts and also, of those of the WZ Sge subgroup which exhibit only superoutbursts (Osaki 1995). Moreover the eccentric, precessing disk naturally explains the superhump periods (e.g. Hirose \& Osaki 1993; Molnar \& Kobulnicky 1992; Whitehurst \& King 1991).

\subsection{PROBLEMS}

The two models predict very different variations of the disk radius and of the lengths of normal cycles within a supercycle (Ichikawa, Hirose \& Osaki 1993; Smak 1991). Unfortunately, the observational evidence, which seems to favor marginally the EMT model, is not adequate for testing these predictions in a decisive way.

Both models have their weak points. The EMT model requires an eccentric, precessing disk to account for superhumps but does not explain why it should become eccentric to begin with. The TTI model, while offering a convincing mechanism for an eccentricity to appear, encounters problems related to its growth. Osaki (1989) and Hirose \& Osaki (1990) maintain that the tidal instability is intrinsic to the disk itself, and that the eccentricity can grow to a finite value within a day or two after the critical disk radius has been exceeded. On the other hand, Whitehurst (1988) and Whitehurst \& King (1991) argue that a strong disturbance by the enhanced mass transfer is needed to accelerate the, otherwise too slow, growth of the eccentricity. The role of the enhanced mass transfer is, however, more complex. Lubow (1994) showed that (i) with variable $\dot{M}$ the stream can indeed induce an eccentricity but it is likely to be small, but (ii) when $\dot{M} \approx$ constant, the stream causes the eccentricity to decrease. Furthermore, during the phase of enhanced mass transfer the radius of the disk contracts and becomes much smaller than the critical radius at which the tidal instability can develop (Ichikawa et al. 1993; Smak 1991).

Perhaps the weakest point of the TTI model is connected with the sequence of events responsible for superoutbursts and superhumps. In the TTI model it begins with a tidal instability, leading to the formation of an eccentric disk and causing a major enhancement of the accretion rate. 
Accordingly, the superhumps should appear at the very early phase of a superoutburst. In most cases, however, they appear one or two days after the superoutburst maximum.

\subsection{A HYBRID EMT + TTI MODEL?}

As the two models appear to complement each other in many respects it is tempting to consider a hybrid model, with a possible scenario which could look like this:

(i) the basic mechanism of a superoutburst and, in particular, the clock controlling the length of a supercycle is that of the EMT model;

(ii) the superoutburst begins with a major enhancement of the mass transfer rate caused by irradiation of the secondary during the preceding normal outburst (which acts as a trigger). At this phase, due to a high $\dot{M}$, the disk contracts and its outer parts become geometrically much thicker;

(iii) during the subsequent phase, due to decreasing irradiation of the secondary caused by a more effective screening by the disk, the mass transfer rate declines;

(iv) at this phase the disk expands considerably (Ichikawa et al. 1993; Smak 1991) and becomes vulnerable to the tidal instability leading to the development of an eccentric, precessing disk. It is then at this phase (but not earlier!) that the superhumps appear;

(v) as both models appear to offer plausible mechanisms for the origin of superhumps, it is not unlikely that a combination of the two mechanisms might provide the best explanation.

Note added after the Colloquium. Jean-Pierre Lasota, in his review talk, brought to the attention of those present at the Colloquium a very important fact concerning the occurence of superoutbursts (which, strangely enough, remained largely unknown!): In 1985 October/November U Gem underwent a superoutburst lasting for $45 \mathrm{~d}$ (Mason et al. 1988). For the models of superoutbursts, which were tailored specifically to explain their occurence exclusively in the very short period, very low mass ratio dwarf novae of the SU UMa subtype, the superoutburst of U Gem (unless it can be explained as a completely different kind of event) implies that all of them may actually be wrong!

\section{References}

Brett, J.M., Smith, R.C., 1993, MNRAS, 264, 641

Cannizzo, J.K., 1993, in "Accretion Disks in Compact Stellar Systems", ed. J.C. Wheeler, Singapore: World Scientific Publishing, p6

Cannizzo, J.K., Shafter, A.W., Wheeler, J.C., 1988, Ap. J., 333, 227

Echevarria, J., Diego, F., Tapia, M., et al., 1989, MNRAS, 240, 975 
Gicger, A., 1987, Acta Astr., 37, 29

Hameury, J.M., King, A.R., Lasota, J.-P. 1993, in "Accretion Disks in Compact Stellar Systems", ed. J.C. Wheeler, Singapore: World Scientific Publishing, p360

Hessman, F.V., Robinson, E.L., Nather, R.E., Zhang, E.-H., 1984, Ap. J., 286, 747

Hirose, M., Osaki, Y., 1990, PASJ, 42, 135

Hirose, M., Osaki, Y., 1993, PASJ, 45, 595

Howell, S.B., Szkody, P., Cannizzo, J.K., 1995, Ap. J., 439, 337

Huang, M., Sion, E.M., Szkody, P., 1995, AJ, 109, 1746

Ichikawa, S., Hirose, M., Osaki, Y., 1993, PASJ, 45, 243

Livio, M., Spruit, H.C., 1991, A\&A, 252, 189

Lubow, S.H., 1994, Ap. J., 432, 224

Marsh, T.R., Horne, K., 1990, Ap. J., 349, 593

Marsh, T.R., Horne, K., Cheng, F., 1993, in "Cataclysmic Variables and Related Physics", eds O. Regev, G. Shaviv, Ann. Israel Phys. Soc., 10, 7

Martin, J.S., Friend, M.T., Smith, R.C., Jones, D.H.P., 1989, MNRAS, 240, 519

Mason, K.O., Cordova, F.A., Watson, M.G., King, A.R., 1988, MNRAS, 232, 779

Meyer, F., Meyer-Hofmeister, E., 1983, A\&A, 121, 29

Mineshige, S., 1988, Ap. J., 335, 881

Molnar, L., Kobulnicky, H.A., 1992, Ap. J., 392, 678

O'Donoghue, D., 1986, MNRAS, 220, 23P

Osaki, Y., 1985, A\&A, 144, 369

Osaki, Y., 1989, PASJ, 41, 1005

Osaki, Y., 1995, PASJ, 47, 47

Osaki, Y., Hirose, M., Ichikawa, S., 1993, in "Accretion Disks in Compact Stellar Systems", ed. J.C. Wheeler, Singapore: World Scientific Publishing, p272

Paczyński, B., 1965, Acta Astr., 15, 305

Paczyński, B., Sienkiewicz, R., 1983, Ap. J., 268, 825

Sarna, M., 1990, A\&A, 239, 163

Smak, J., 1984a, Acta Astr., 34, 93

Smak, J., 1984b, Acta Astr., 34, 161

Smak, J., 1987, A\&SS, 131, 497

Smak, J., 1991, Acta Astr., 41, 269

Smak, J., 1993, Acta Astr., 43, 101

Smak, J., 1995, Acta Astr., 45, 355

Sparks, W.M. 1996, private communication

Sparks, W.M., Sion, E.M., Starrfield, S.G., Austin, S., 1993, in "Cataclysmic Variables and Related Physics", eds O. Regev, G. Shaviv, Ann. Israel Phys. Soc., 10, 96

Vogt, N., 1981, Ap. J., 252, 653

Vogt, N., 1983, A\&A, 118, 95

Vogt, N., 1993, in "Cataclysmic Variables and Related Physics", eds O. Regev, G. Shaviv, Ann. Israel Phys. Soc., 10,63

Warner, B., 1987, MNRAS, 227, 23

Warner, B., 1995, Cataclysmic Variable Stars, Cambridge: Cambridge Univ.Press.

Warner, B., O'Donoghue, D., 1988, MNRAS, 233, 705

Whitehurst, R., 1988, MNRAS, 232, 35

Whitehurst, R., King, A.R., 1991, MNRAS, 249, 25

Wolf, S., Mantel, K.H., Horne, K., et al., 1993, A\&A, 273, 160

Wood, J.H., Marsh, T.R., Robinson, E.L., et al., 1989, MNRAS, 239, 809

Wood, J.H., Robinson, E.L., Bless, R.C., et al., 1993, in "Cataclysmic Variables and Related Physics", eds O. Regev, G. Shaviv, Ann. Israel Phys. Soc., 10, 19

Zoła, S., 1989, Acta Astr., 39, 45 